\title{
Retraction Note: Aberrant mannosylation profile and FTX/miR-342/ALG3-axis contribute to development of drug resistance in acute myeloid leukemia
}

\author{
Bing Liu, Xiaolu Ma, Qianqian Liu, Yang Xiao, Shimeng Pan and Li Jia(i)
}

Retraction to: Cell Death \& Disease https://doi.org/10.1038/s41419-018-0706-7

published online 07 June 2018

The Editors-in-Chief have retracted this article because there appears to be replication between a group of data points in the $10.95 \%$ quadrant of the U/A (VCR) ALG3 shRNA panel of Fig. 3f and a group of data points in the $4.52 \%$ quadrant of the T/A (Paxcitaxel) ALG3 shRNA panel of Fig. 3f. The Editors-in-Chief therefore no longer have confidence in the integrity of the data in Fig. 3. All of the authors disagree with this retraction.

Published online: 12 February 2020 